ORIGINAL ARTICLE

\title{
Utility of overnight pulse oximetry and heart rate variability analysis to screen for sleep-disordered breathing in chronic heart failure
}

\author{
Neil R Ward, ${ }^{1}$ Martin R Cowie, ${ }^{2}$ Stuart D Rosen, ${ }^{2,3}$ Vitor Roldao, ${ }^{1}$ Manuel De Villa, ${ }^{2}$ \\ Theresa A McDonagh, ${ }^{2}$ Anita Simonds, ${ }^{1}$ Mary J Morrell ${ }^{1}$
}

\begin{abstract}
- Additional materials are published online only. To view these files please visit the journal online (http://dx.doi.org/ 10.1136/thoraxjnl-2012201684).

${ }^{1}$ Clinical and Academic Department of Sleep and Breathing, National Heart and Lung Institute and NIHR Biomedical Research Unit, Royal Brompton Hospital and Imperial College, London, UK

${ }^{2}$ Department of Cardiac Medicine, National Heart and Lung Institute, Royal Brompton Hospital and Imperial College, London, UK

${ }^{3}$ Ealing Hospital NHS Trust, Southall, UK
\end{abstract}

\section{Correspondence to}

Dr Neil R Ward, Academic Unit of Sleep and Breathing, Royal Brompton Hospital, London SW3 6NP, UK:

neil.ward@nhs.net

Received 27 January 2012 Accepted 19 June 2012

Published Online First

17 July 2012

\section{ABSTRACT}

Background Sleep-disordered breathing (SDB) is under diagnosed in chronic heart failure (CHF). Screening with simple monitors may increase detection of SDB in a cardiology setting. This study aimed to evaluate the accuracy of heart rate variability analysis and overnight pulse oximetry for diagnosis of SDB in patients with CHF. Methods 180 patients with CHF underwent simultaneous polysomnography, ambulatory electrocardiography and wrist-worn overnight pulse oximetry. SDB was defined as an apnoea-hypopnoea index $\geq 15 / h$. To identify SDB from the screening tests, the per cent very low frequency increment (\%VLFI) component of heart rate variability was measured with a pre-specified cutoff $\geq 2.23 \%$, and the $3 \%$ oxygen desaturation index was measured with a pre-specified cutoff $>7.5$ desaturations/h.

Results 173 patients with CHF had adequate sleep study data; SDB occurred in 77 (45\%) patients. Heart rate variability was measurable in $78(45 \%)$ patients with area under the \%VLFI receiver operating characteristic curve of 0.50 . At the $\geq 2.23 \%$ cutoff, $\%$ VLFI sensitivity was $58 \%$ and specificity was $48 \%$. The $3 \%$ oxygen desaturation index was measurable in $171(99 \%)$ patients with area under the curve of 0.92 . At the pre-specified cutoff of $>7.5$ desaturations/h, the $3 \%$ oxygen desaturation index had a sensitivity of $97 \%$, specificity of $32 \%$, negative likelihood ratio of 0.08 and positive likelihood ratio of 1.42 . Diagnostic accuracy was increased using a cutoff of 12.5 desaturations/h, with sensitivity of $93 \%$ and specificity of $73 \%$.

Conclusions The high sensitivity and low negative likelihood ratio of the 3\% oxygen desaturation index indicates that pulse oximetry would be of use as a simple screening test to rule out SDB in patients with CHF in a cardiology setting. The \%VLFI component of heart rate variability is not suitable for detection of SDB in CHF.

\section{INTRODUCTION}

Sleep-disordered breathing (SDB) occurs in $\geq 50 \%$ of patients with chronic heart failure (CHF) ${ }^{12}$ and despite an independent association with increased mortality, ${ }^{34}$ it is under diagnosed. ${ }^{5}$ Identification of patients with CHF who have SDB is complicated by the absence of classic symptoms such as excessive daytime sleepiness. ${ }^{6}$ Additionally, polysomnography is recommended for the diagnosis of $\mathrm{SDB}$ in $\mathrm{CHF}^{7}$ but these patients are often seen in
Key messages

What is the key question?

- Can analysis of heart rate variability or overnight pulse oximetry be used to detect sleepdisordered breathing (SDB) in patients with chronic heart failure (CHF)?

What is the bottom line?

- Overnight pulse oximetry has a high diagnostic accuracy for SDB in CHF. Analysis of heart rate variability is not suitable to identify patients with CHF who have SDB.

\section{Why read on?}

- This study shows that overnight pulse oximetry can be used to diagnose SDB in patients with CHF. Depending on the diagnostic cutoff chosen, pulse oximetry may be of greatest clinical value as a 'rule out' test.

cardiology clinics and access to this investigation may be limited. There is a need for simpler methods to enable the screening of patients with CHF for SDB.

Portable monitors such as overnight pulse oximetry have been used to diagnose obstructive sleep apnoea (OSA) in otherwise healthy adults but their utility in patients with comorbid medical conditions is unclear. ${ }^{8}$ A recent international workshop report has highlighted the need for further research to evaluate the role of portable monitors in patients with SDB and CHF. ${ }^{9}$

We have previously shown in a small study that analysis of heart rate variability (HRV) can be used to rule out SDB in patients with $\mathrm{CHF}^{10} \mathrm{HRV}$ analysis can be performed during cardiological assessment and is potentially a convenient method to screen for SDB in CHF. The primary aim of this study was to prospectively evaluate the diagnostic performance of HRV analysis to detect SDB in a large number of patients with CHF in a general cardiology setting. HRV analysis may not be possible in patients with cardiac arrhythmias or paced cardiac rhythm and our secondary aim was to evaluate pulse oximetry as a screening strategy for patients with CHF who are unsuitable for HRV analysis. The following hypotheses were tested: 
HRV analysis can be used to rule out SDB in patients with CHF; overnight pulse oximetry can be used to rule out SDB in patients with CHF and atrial fibrillation, paced cardiac rhythm, frequent ectopic beats or artefact on ambulatory ECG monitoring; HRV analysis has a higher accuracy than overnight pulse oximetry for the diagnosis of SDB in CHF.

Some of the results of this study have previously been presented in abstract form. ${ }^{11} 12$

\section{METHODS}

Patients with low or preserved ejection fraction heart failure were recruited from cardiology outpatient clinics. Inclusion criteria required a diagnosis of CHF in accordance with European guidelines, ${ }^{13}$ no hospitalisation or change in medication for $\geq 4$ weeks and age 18-90 years. Patients receiving treatment for $\mathrm{SDB}$ were excluded. Eligible patients were invited to participate irrespective of clinical suspicion of SDB.

All patients underwent unattended polysomnography (SOMNOscreen, SOMNOmedics, Randersacker, Germany) with additional simultaneous recording with ambulatory electrocardiography (VistaPlus, Novacor, Rueil Malmaison Cedex, France) and wrist worn pulse oximetry (Pulsox 3i, Konica Minolta, Tokyo, Japan) on the same night. Patients were studied in their own home or in hospital according to their preference. Anthropometric measurements and clinical interview were completed before the sleep study with a behavioural maintenance of wakefulness test (Oxford sleep resistance test (OSLER)) performed the next morning. ${ }^{14}$ Full details of measurements are provided in the online supplement.

Polysomnography studies were analysed by one person unaware of the clinical status of the patient, using standard scoring criteria. ${ }^{15}$ Apnoea was scored when nasal airflow reduced to $<10 \%$ of baseline for $\geq 10 \mathrm{~s}$. Hypopnoea was scored when nasal airflow reduced by $\geq 50 \%$ of baseline with a $\geq 3 \%$ oxygen desaturation and/or electroencephalographic arousal (see also online supplement). SDB was defined as an apnoea-hypopnoea index $(\mathrm{AHI}) \geq 15 / \mathrm{h}$ and was categorised as central sleep apnoea (CSA) or OSA.

The ambulatory ECG was recorded with a sampling frequency of $200 \mathrm{~Hz}$. HRV was analysed (Holtersoft, Novacor) during the period from 00:00 to 06:00. Patients with paced cardiac rhythm, atrial fibrillation or $>10 \%$ ectopic beats were excluded from HRV analysis. Manual editing was performed to ensure all ORS complexes were correctly identified. The spectral density of the heart rate increment was analysed for measurement of the per cent very low frequency increment (\%VLFI). ${ }^{16}$ This component represents the very low frequency power $(0.01-0.05 \mathrm{~Hz})$ of the heart rate increment power expressed as the percentage of total power $(0.01-0.5 \mathrm{~Hz})$. An a priori \%VLFI cut-off of $\geq 2.23 \%$ was used to diagnose SDB. ${ }^{10}$ Recommended time domain and spectral components of HRV were also measured. ${ }^{17}$

The wrist worn pulse oximeter recorded oxygen saturation with a sampling time of $5 \mathrm{~s}$ and data storage frequency of $0.2 \mathrm{~Hz}$. Overnight pulse oximetry was analysed (Download 2001, Stowood Scientific Instruments, Oxford, UK) for $\geq 3 \%$ oxygen desaturations during the 'time in bed' period recorded by the patient. The $3 \%$ oxygen desaturation index ( $3 \%$ ODI) was calculated as the mean number of $\geq 3 \%$ oxygen desaturations per hour, using the 'time in bed' as denominator. A pre-specified $3 \%$ ODI cutoff of $>7.5$ desaturations/h was used to diagnose SDB.

\section{Statistical analyses}

An a priori sample size of 180 patients with CHF was calculated to be adequately powered $(1-\beta=80 \%)$ to detect a one-sided difference of $>10 \%$ between the sensitivity/specificity of HRV and polysomnography, at a significance level $(\alpha)$ of 0.05 (see online supplement). Results are presented as median and IOR. Continuous variables were compared with Mann-Whitney or Kruskal-Wallis tests. Categorical variables were compared using Fisher's exact test or $\chi^{2}$ test. The diagnostic accuracy of the $\%$ VLFI and $3 \%$ ODI were compared by measurement of the area under the receiver-operating characteristic (ROC) curve. Sensitivity, specificity, predictive values and likelihood ratios for the $\%$ VLFI and $3 \%$ ODI were determined at the a priori cutoffs. Statistical analyses were performed using SPSS V.16.0.

\section{RESULTS}

Three hundred and fifty-four patients with CHF were identified who fulfilled the recruitment criteria, 180 of whom consented to participate (figure 1). One hundred and seventy-three patients with adequate polysomnography data were included in the analysis, with median age 69.8 (58.8-76.8) years and $86 \%$ were men (table 1). Most patients had mild to moderate symptomatic CHF with New York Heart Association class I or II symptoms in $77 \%$ and median left ventricle ejection fraction 40\% (28-59\%). One hundred and thirty-two (73\%) patients had overnight monitoring performed in their home.

\section{Diagnosis of SDB from polysomnography}

The median AHI was 13.5 (7.2-24.8) events/h. SDB was diagnosed in $77(45 \%)$ patients with CHF, with OSA in $53(31 \%)$ and CSA in 24 (14\%). As only a small number of patients had CSA, all patients with SDB were analysed as a single group. Body mass index, neck circumference and hip:waist ratio were significantly higher in patients with CHF who had SDB

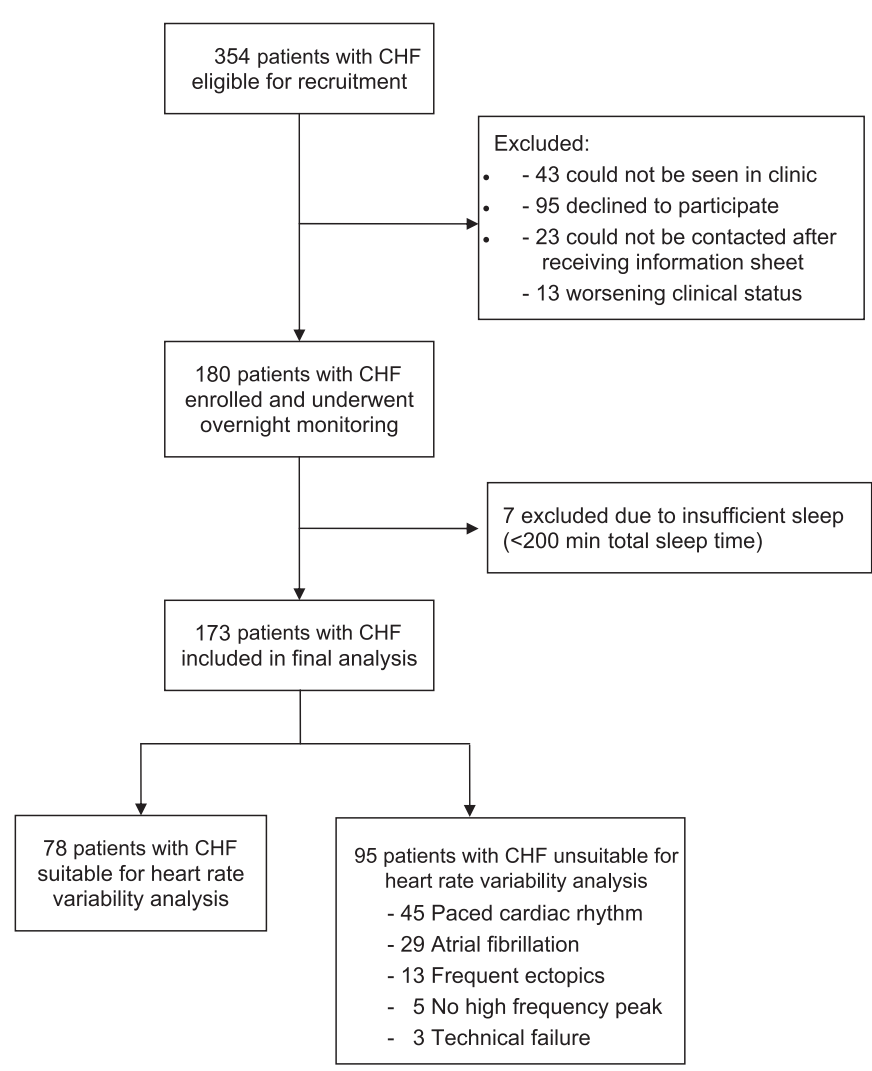

Figure 1 Flow diagram to show recruitment of patients with chronic heart failure (CHF) and inclusion in heart rate variability analysis. 
Table 1 Clinical characteristics of patients with chronic heart failure $(n=173)$

\begin{tabular}{|c|c|}
\hline Clinical characteristic & Value \\
\hline Age (years) & $69.8(58.8-76.8)$ \\
\hline Men $(n, \%)$ & $148(85.5)$ \\
\hline BMI $\left(\mathrm{kg} / \mathrm{m}^{2}\right)$ & $29.1(25.4-32.7)$ \\
\hline Neck size $(\mathrm{cm})$ & $40.0(38.0-43.0)$ \\
\hline Waist/hip ratio & $1.02(0.98-1.06)$ \\
\hline Diabetes (n, \%) & $44(25)$ \\
\hline COPD/asthma (n, \%) & $34(20)$ \\
\hline Ischaemic cardiomyopathy (n, \%) & $89(51)$ \\
\hline$\beta$ blocker therapy (n, \%) & $126(73)$ \\
\hline ACEI/ARB therapy $(\mathrm{n}, \%)$ & $157(91)$ \\
\hline Atrial fibrillation (n, \%) & $52(30)$ \\
\hline Cardiac pacing (n, \%) & $50(29)$ \\
\hline NYHA classification (I/II/III/IV) & $24 / 110 / 37 / 2$ \\
\hline $\mathrm{BNP}(\mathrm{pg} / \mathrm{ml})$ & $118(55-239)$ \\
\hline Left ventricular ejection fraction (\%) & $40(28-58)$ \\
\hline
\end{tabular}

(table 2). There were no significant differences in New York Heart Association class, brain natriuretic peptide or left ventricle ejection fraction among patients with and without SDB. Subjective and objective measures of sleepiness were similar irrespective of the presence or absence of SDB, but patients with SDB had significantly more stage 1 sleep and higher arousal index (table 3).

\section{Diagnostic accuracy of HRV}

HRV was measurable in 78 (45\%) patients with CHF; the most common reasons for unsuitability for HRV measurement were paced cardiac rhythm or atrial fibrillation in 74 (43\%) patients. Patients in whom HRV was measurable were significantly younger $(p=0.006)$ with a lower median brain natriuretic peptide $(p<0.001)$ and a trend to higher left ventricle ejection

Table 2 Comparison of clinical characteristics in patients with chronic heart failure with or without sleep-disordered breathing

\begin{tabular}{lccc}
\hline & No SDB (n=96) & SDB (n=77) & p Value \\
\hline Age (years) & $71.9(59.4-77.6)$ & $66.7(57.1-75.0)$ & 0.08 \\
Men (n, \%) & $81(84)$ & $67(87)$ & 0.67 \\
BMI $\left(\mathrm{kg} / \mathrm{m}^{2}\right)$ & $27.4(24.8-31.6)$ & $30.8(27.8-35.4)$ & $<0.001$ \\
Neck size (cm) & $39.0(37.0-41.0)$ & $42.0(39.5-43.0)$ & $<0.001$ \\
Waist/hip ratio & $1.01(0.96-1.05)$ & $1.03(0.99-1.07)$ & 0.04 \\
Diabetes (n, \%) & $22(23)$ & $22(29)$ & 0.48 \\
COPD/asthma (n, \%) & $27(28)$ & $7(9)$ & 0.002 \\
Ischaemic cardiomyopathy & $54(56)$ & $35(46)$ & 0.17 \\
(n, \%) & & & \\
$\beta$ & $62(65)$ & $64(83)$ & 0.009 \\
AClocker therapy (n, \%) & $87(91)$ & $70(91)$ & 1.0 \\
Atrial fibrillation (n, \%) & $26(27)$ & $26(34)$ & 0.41 \\
Cardiac pacing (n, \%) & $25(26)$ & $26(34)$ & 0.32 \\
NYHA classification (I/ll/ll//V) & $12 / 64 / 20 / 0$ & $12 / 46 / 17 / 2$ & 0.37 \\
BNP (pg/ml) & $128(69-201)$ & $107(42-322)$ & 0.97 \\
Left ventricle ejection & $40(28-58)$ & $43(32-58)$ & 0.76 \\
fraction (\%) & & & \\
\hline
\end{tabular}

Data are presented as median (IQR), or number (\%) of patients.

ACEI/ARB, ACE inhibitor or angiotensin receptor blocker; BMI, body mass index; BNP

B-type natriuretic peptide; COPD, chronic obstructive pulmonary disease; NYHA, New York Heart Association; SDB, sleep-disordered breathing.
Table 3 Comparison of sleepiness and measurements of sleep in patients with chronic heart failure with and without sleep-disordered breathing

\begin{tabular}{lccc}
\hline & No SDB $(\mathbf{n}=\mathbf{9 6})$ & SDB $(\mathbf{n}=\mathbf{7 7})$ & p Value \\
\hline Epworth score & $7(4-10)$ & $7(5-10)$ & 0.45 \\
OSLER duration (min) & $40(39-40)$ & $40(27-40)$ & 0.18 \\
HADS score & $12(6-17)$ & $11(6-16)$ & 0.78 \\
Total sleep time (min) & $355(285-401)$ & $351(295-408)$ & 0.55 \\
Stage 1 sleep (\% TST) & $25(14-42)$ & $32(21-48)$ & 0.02 \\
Stage 2 sleep (\% TST) & $39(27-52)$ & $38(21-46)$ & 0.15 \\
Deep sleep (\% TST) & $11(6-18)$ & $10(6-17)$ & 0.73 \\
REM sleep (\% TST) & $19(15-25)$ & $17(13-24)$ & 0.41 \\
Sleep efficiency (\%) & $69(61-80)$ & $74(63-81)$ & 0.26 \\
Arousal index (events/h) & $16(13-22)$ & $24(19-36)$ & $<0.001$ \\
AHI (events/h) & $7.6(4.1-11.0)$ & $27.0(19.2-40.6)$ & \\
Time Sp0 $0_{2}<90 \%$ (\% TST) & $1.1(0.1-19.8)$ & $8.3(1.8-22.7)$ & $<0.001$ \\
Baseline Sp0 $0_{2}(\%)$ & $93(91-95)$ & $94(92-95)$ & 0.62 \\
3\% 0Dl* (events/h) & $9.1(6.2-13.0)$ & $24.4(17.9-37.7)$ & $<0.001$ \\
Periodic limb movement & $14(2-33)$ & $11(3-42)$ & 0.78 \\
index (events/h) & & & \\
\hline
\end{tabular}

Data are presented as median (IQR).

* $3 \%$ ODI measured from wrist worn pulse oximeter.

AHI, apnoea-hypopnoea index; HADS, Hospital Anxiety and Depression score; ODI, oxygen desaturation index; OSLER, Oxford Sleep Resistance Test; REM, rapid eye movement; $\mathrm{SpO}_{2}$, oxygen saturation; TST, total sleep time.

fraction $(p=0.08)$ compared with those in whom HRV could not be measured (table E1 in online supplement).

In the 78 (45\%) patients with CHF in whom HRV was measurable, SDB occurred in $36(46 \%)$. The \%VLFI component of HRV had a poor diagnostic accuracy for detection of SDB with an area under the \%VLFI ROC curve of 0.50 (95\% CI 0.37 to $0.63, p=0.99$ ) (figure 2 ). At the a priori cutoff of $\geq 2.23 \%$, the $\%$ VLFI had a sensitivity of $58 \%$, specificity of $48 \%$, positive likelihood ratio of 1.11 and negative likelihood ratio of 0.88 for diagnosis of SDB (table E2).

The \%VLFI was not correlated with the AHI (Spearman's $\rho$ 0.005 ) and there was no significant difference in \%VLFI according to the presence or absence of SDB (median \%VLFI: patients without SDB, $2.46 \%$ (1.45-4.41); patients with SDB,

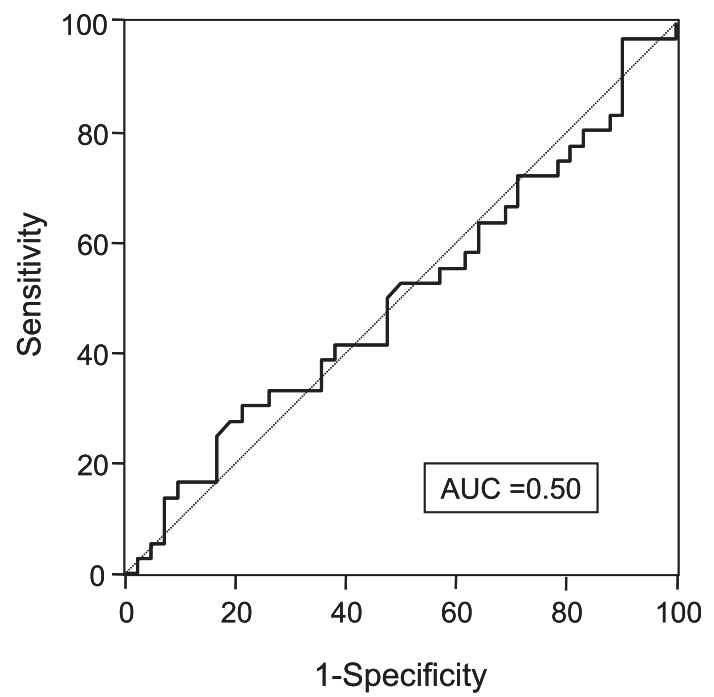

Figure 2 Per cent very low frequency increment receiver-operating characteristic curve for diagnosis of sleep-disordered breathing (apnoeahypopnoea index $\geq 15 / h$ ) in patients with chronic heart failure. AUC, area under curve. 

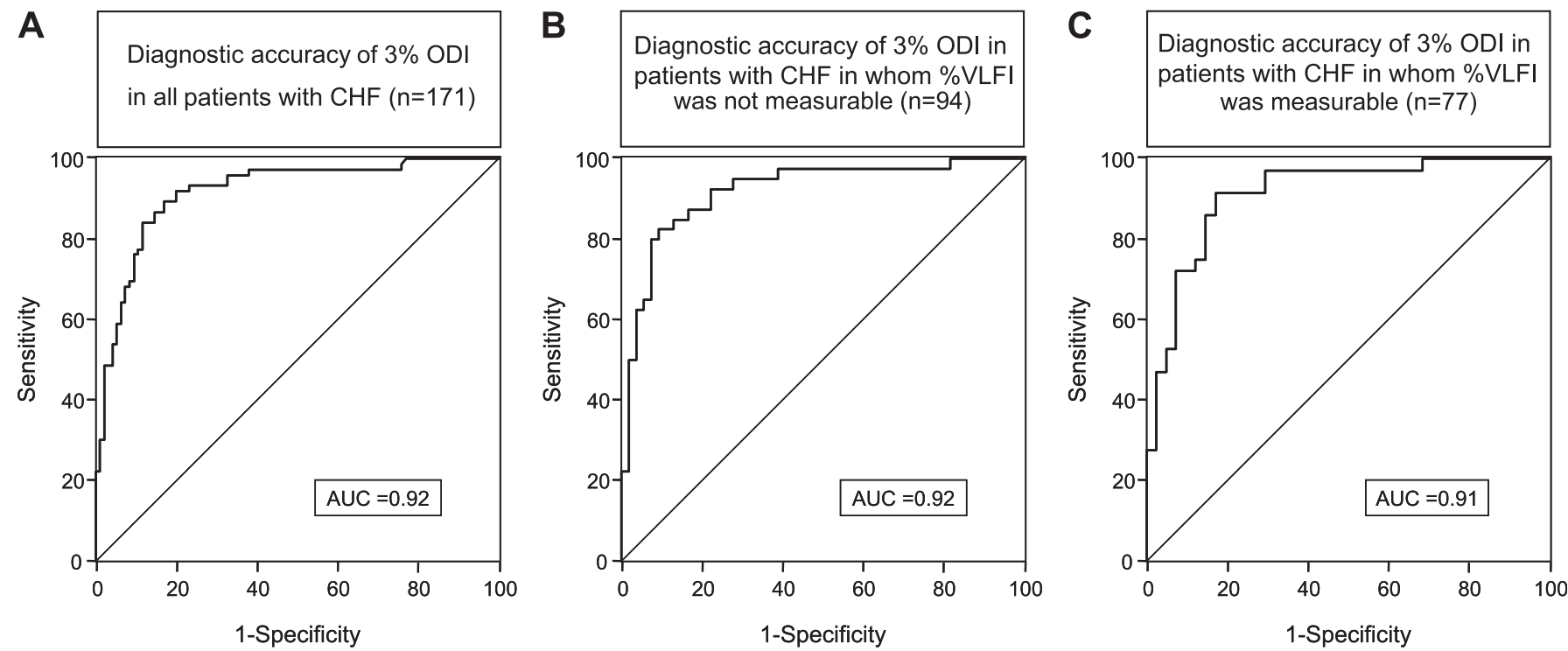

Figure 3 Receiver-operating characteristic curves showing diagnostic accuracy of $3 \%$ oxygen desaturation index (3\% ODI) for diagnosis of sleepdisordered breathing in (A) all patients with chronic heart failure (CHF) $(n=171)$; (B) patients with CHF in whom per cent very low frequency increment (\%VLFI) could not be measured ( $n=94)$; and (C) patients with CHF in whom \%VLFI was measured $(n=77)$. AUC, area under curve.

$2.32 \%(1.19-5.08), \mathrm{p}=0.99)$. SDB was categorised as OSA in 31 (40\%) patients with median \%VLFI $2.35 \%(1.35-5.41)$; in $5(6 \%)$ patients with CSA, median \%VLFI was $0.78 \%$ (0.58-4.18). There was no significant difference between \%VLFI in patients with CHF with OSA, CSA or without SDB ( $\mathrm{p}=0.39)$.

The diagnostic accuracy of \%VLFI was assessed using alternative AHI thresholds of $\geq 5, \geq 20$ and $\geq 30$ events/h for diagnosis of SDB. The area under the \%VLFI ROC curve was similar at all AHI thresholds with no significant change in sensitivity or specificity (table E2).

\section{Diagnostic accuracy of overnight pulse oximetry}

Overnight pulse oximetry could be analysed in 171 (99\%) patients with CHF in whom SDB was present in $76(44 \%)$. There was good agreement between 3\% ODI and AHI (mean difference $=-0.2$ events $/$ h; figure E1) with correlation coefficient (Spearman's $\rho$ ) of 0.84 (figure E2). The 3\% ODI had an area under the ROC curve of 0.92 ( $95 \%$ CI 0.88 to 0.96 ) for detection of SDB in CHF, indicating high diagnostic accuracy (figure $3 \mathrm{~A}$ ). At the a priori cutoff of $>7.5$ desaturations/h for diagnosis of $\mathrm{SDB}$ in CHF, two false-negative results occurred with a sensitivity of $97 \%$ and negative likelihood ratio of 0.08 (table 4). However, at this cutoff there were 65 false-positive results with a specificity of $32 \%$ and a positive likelihood ratio of 1.42 . In a post hoc analysis, a $3 \%$ ODI cutoff of $>12.5$ desaturations $/ \mathrm{h}$ reduced the number of false-negative oximetry studies to 26 , specificity increased to $73 \%$ (95\% CI $64 \%$ to $81 \%$ ), sensitivity was $93 \%$ (95\% CI $86 \%$ to $97 \%$ ) and negative likelihood ratio was 0.09 (95\% CI 0.04 to 0.21 ).

To investigate the utility of overnight pulse oximetry for diagnosis of SDB in patients with CHF who were unsuitable for HRV analysis, the diagnostic accuracy of the 3\% ODI was assessed in 94 patients in whom \%VLFI was not measurable. In this subgroup, the 3\% ODI had area under the ROC curve of 0.92 ( $95 \%$ CI 0.86 to 0.98 ; figure $3 \mathrm{~B}$ ). At the cutoff of $>7.5$ desaturations/h, the $3 \%$ ODI had a sensitivity of $98 \%$, specificity of $24 \%$, positive likelihood ratio of 1.28 and negative likelihood ratio of 0.10 for diagnosis of SDB in patients with $\mathrm{CHF}$ who were unsuitable for HRV analysis (table 4).

\section{Diagnostic accuracy of HRV analysis versus overnight pulse oximetry}

To compare HRV analysis and overnight pulse oximetry for detection of SDB in CHF, the diagnostic accuracy of the 3\% ODI was assessed in 77 patients in whom \%VLFI was measurable (table 4). In this subgroup, the 3\% ODI had area under the ROC curve of 0.91 ( $95 \%$ CI 0.85 to 0.98 ; figure $3 \mathrm{C}$ ) which was significantly greater than the value for \%VLFI $(p<0.0001)$.

\section{DISCUSSION}

The main findings of this study were that the \%VLFI component of HRV was unable to discriminate between the presence and absence of SDB in patients with CHF. Moreover, it could be measured in less than half of the patients. In contrast, the $3 \%$

Table 4 Diagnostic accuracy of 3\% oxygen desaturation index for detection of sleep-disordered breathing in patients with chronic heart failure

\begin{tabular}{|c|c|c|c|c|c|c|c|}
\hline & Sensitivity & Specificity & $\begin{array}{l}\text { Positive } \\
\text { predictive value }\end{array}$ & $\begin{array}{l}\text { Negative } \\
\text { predictive value }\end{array}$ & $\begin{array}{l}\text { Positive } \\
\text { likelihood ratio }\end{array}$ & $\begin{array}{l}\text { Negative } \\
\text { likelihood ratio }\end{array}$ & $\begin{array}{l}\text { Area under } \\
\text { ROC curve }\end{array}$ \\
\hline All patients with $\mathrm{CHF}^{*}(\mathrm{n}=171)$ & $97(91-100)$ & $32(22-41)$ & $53(45-62)$ & 94 (79-99) & $1.42(1.24-1.64)$ & $0.08(0.02-0.34)$ & $0.92(0.88-0.96)$ \\
\hline $\begin{array}{l}\text { Patients with CHF without \%VLFI } \\
\text { measurement* }(n=94)\end{array}$ & $98(87-100)$ & $24(13-35)$ & $49(38-60)$ & $93(66-100)$ & $1.28(1.10-1.50)$ & $0.10(0.01-0.76)$ & $0.92(0.86-0.98)$ \\
\hline Patients with CHF with \%VLFI & $97(85-100)$ & $41(26-57)$ & $59(47-72)$ & $94(73-100)$ & $1.66(1.28-2.16)$ & $0.07(0.01-0.48)$ & $0.91(0.85-0.98)$ \\
\hline
\end{tabular}

measurement* $(n=77)$

*Diagnostic accuracy values were assessed using the a priori cutoff of $>7.5$ desaturations/h for diagnosis of sleep-disordered breathing and are presented with $95 \% \mathrm{Cl}$.

CHF, chronic heart failure; ROC, receiver operating characteristic; \%VLFI, \% very low frequency increment of heart rate variability. 
ODI was measurable in $99 \%$ of patients with CHF, with an area under the ROC curve of 0.92 , indicating high accuracy for diagnosis of SDB. The $3 \%$ ODI had a superior diagnostic accuracy to the \%VLFI for detection of SDB in CHF.

Portable monitors provide a cost-effective approach to improve access to OSA testing. ${ }^{18}$ The simplest portable monitors record one or two physiological parameters with high accuracy for diagnosis of OSA in otherwise healthy adults. ${ }^{19} 20$ The utility of these monitors to diagnose SDB in patients with comorbid cardiovascular disease has been identified as a research priority. ${ }^{9}$ The findings of the present study help address this evidence gap.

HRV analysis has been proposed as a simple screening test for detection of OSA. ${ }^{21} 22$ The occurrence of SDB in CHF increases $\mathrm{HRV}^{23}$ with increased very low frequency $(<0.04 \mathrm{~Hz})$ oscillations in heart rate occurring during $\mathrm{CSA}^{23} 24$ or OSA. ${ }^{23} 25$ In individuals without CHF, the \%VLFI has a sensitivity of $78-91 \%$ and specificity of $34-70 \%$ for diagnosis of OSA. ${ }^{26} 27$ In our previous study of 33 patients with CHF in whom HRV was measurable, the \%VLFI had a sensitivity of $85 \%$ and specificity of $65 \%$ for diagnosis of SDB at an optimum cutoff of $\geq 2.23 \%$. ${ }^{10}$ The current study was designed to be adequately powered to prospectively evaluate this cutoff and has shown that the \% VLFI cannot be used to identify SDB in CHF. It is likely that the larger sample size and greater statistical power of the current study have provided a more accurate measure of the diagnostic utility of \%VLFI in patients with CHF, highlighting the need for adequately powered trials in this area. ${ }^{9}$ Moreover, our findings concur with those of Damy et al, ${ }^{28}$ who have also reported that $\%$ VLFI has a poor accuracy for SDB diagnosis in 54 patients with CHF.

Potential reasons for the absence of a correlation between the $\%$ VLFI and AHI in patients with CHF include a reduction in HRV due to impaired autonomic cardiac reactivity. ${ }^{29}$ Cardiac autonomic regulation and spectral components of HRV are significantly reduced by ageing ${ }^{30}$ and the median age of patients in this study was higher than previous studies. Comorbidities such as diabetes, present in $26 \%$ of patients in this study, may have impaired autonomic function and reduced HRV. Cardiovascular medications are also likely to have influenced HRV in this study as $\beta$ blockers and angiotensin-converting enzyme inhibitors can increase the high-frequency component of HRV through modulation of vagal cardiac input. ${ }^{31} 32$

Concomitant sleep disorders could have influenced any relationship between the \%VLFI and AHI. Periodic limb movements occur frequently in patients with $\mathrm{CHF}^{1}$ and are associated with a significant increase in $\%$ VLFI. ${ }^{26}{ }^{33}$ In the current study, a periodic limb movement index (PLMI) $\geq 5 / \mathrm{h}$ was observed in 111 (64\%) patients with CHF. However, there was no correlation between \%VLFI and PLMI (Spearmans $\rho$ 0.01) and median \% VLFI was not significantly different in patients with PLMI $\geq 5 / \mathrm{h}$ compared with those with PLMI $<5 / \mathrm{h}$. Insomnia and sleep fragmentation unrelated to SDB are also prevalent in $\mathrm{CHF}$ and can increase the \%VLFI. ${ }^{26}$ In the current study, there was no correlation between the \%VLFI and arousal index (Spearmans $\rho$ 0.034). Therefore, we do not think these factors contributed to the present findings.

Overnight pulse oximetry was simultaneously investigated as a simple screening test for the detection of SDB in CHF. Measurement of $\geq 2 \%$ or $\geq 4 \%$ oxygen desaturations has been reported to have high sensitivity to detect SDB in patients with low ejection fraction $\mathrm{CHF}^{34} 35$ In this study, the area under the ROC curve for $3 \%$ ODI indicated a high diagnostic accuracy for detection of SDB in patients with low and preserved ejection fraction heart failure. At the a priori cutoff of $>7.5$ desaturations/h, 3\% ODI had high sensitivity with a clinically useful negative likelihood ratio of 0.08 , and would therefore be of greatest clinical use to rule out SDB in CHF.

If overnight pulse oximetry was used as the initial investigation in a phased testing approach to detect SDB in CHF, further sleep studies would not have been required in 32 (33\%) patients with CHF without SDB. However, at the cutoff of $>7.5$ desaturations/h, 3\% ODI had poor specificity and low positive likelihood ratio. The diagnostic specificity of $3 \%$ ODI was increased at a cutoff of $>12.5$ desaturations/h without reduction in sensitivity. Using the $3 \%$ ODI cutoff of $>12.5$ desaturations $/ h$ would have correctly identified and saved further sleep studies in $69(73 \%)$ patients with CHF without SDB.

A limitation of the present study is that paced cardiac rhythm or atrial fibrillation prevented HRV analysis in $43 \%$ of patients with CHF. While HRV may still be measurable in patients with cardiac resynchronisation therapy who are not atrial paced, and Cheyne-Stokes respiration can increase very low frequency HRV in patients with atrial fibrillation, ${ }^{36}$ the results of this study suggest that this would be of no value to screen for SDB in CHF. A second consideration is that we did not meet our desired sample size as HRV could not be analysed in all 180 patients with CHF recruited. However, further studies of the utility of \% VLFI for diagnosis of SDB in CHF may not be an efficient use of resources; our results indicate a sample size of 780 patients with CHF suitable for HRV analysis would be required to be adequately powered to exclude a $>0.5 \%$ difference in \%VLFI between patients with and without SDB. A third consideration is that the small number of patients with CHF and CSA precluded comparison of the diagnostic accuracy of overnight pulse oximetry between patients with CSA and OSA. However, overnight oximetry has previously been reported to have $100 \%$ sensitivity for CSA in $\mathrm{CHF}^{34}$ and in the current study, there were no false-negative oximetry results in patients with CSA

A strength of this study is the recruitment of a heterogeneous population of patients with CHF from cardiology clinics, $34 \%$ of whom had an ejection fraction $\geq 50 \%$. Many previous studies have only enrolled patients with low ejection fraction heart failure but up to $50 \%$ of patients with $\mathrm{CHF}$ have heart failure with preserved ejection fraction, ${ }^{37}$ and SDB may contribute to cardiac dysfunction in these patients. ${ }^{38}$ In addition, despite inclusion of patients with CHF and comorbidities including COPD and asthma which may influence overnight oxygen saturation, the diagnostic accuracy of the 3\% ODI was high with area under the ROC curve of 0.92 . However, this may have contributed to the low specificity of pulse oximetry at the cutoff of $>7.5$ desaturations $/ \mathrm{h}$.

In summary, in patients with CHF recruited from a cardiology setting, the $3 \%$ ODI cutoff of $>7.5$ desaturations/h had a high sensitivity of $97 \%$ and low negative likelihood ratio of 0.08 for diagnosis of SDB. Specificity of the 3\% ODI was increased to $73 \%$ without reduction in sensitivity at an optimum 3\% ODI cutoff of 12.5 desaturations/h. In contrast, the \%VLFI had a poor diagnostic accuracy and it cannot be recommended to screen for SDB in patients with CHF. Overnight pulse oximetry would be of greatest clinical use to rule out SDB in patients with $\mathrm{CHF}$, helping to reduce pressure on sleep laboratory facilities and prioritise patients who may require more detailed sleep studies.

Acknowledgements The authors are grateful to Dr B North and Dr E Kulinskaya of the Imperial College Statistical Advisory Service for providing statistical support for this study, and to Dr J Hooper of the Clinical Chemistry Department at the Royal Brompton Hospital for assistance with the BNP assay. 
Contributors MRC, SDR, AS, TAM, MJM: conceived the idea for this study. NRW, VR, MDV: data collection and analysis. NRW, MJM, AS, SDR, MRC: interpretation of data. NRW and MJM wrote the manuscript with critical input from SDR, AS and MRC.

Funding This study was funded by a grant from the British Heart Foundation and supported by the NIHR Respiratory Disease Biomedical Research Unit at the Royal Brompton and Harefield NHS Foundation Trust and Imperial College London. Ambulatory electrocardiography recorders and heart rate variability analysis software were provided on loan from the manufacturer, Novacor, Paris, France.

Competing interests None.

Ethics approval Ethics approval was provided by Brompton, Harefield and NHLI Research Ethics Committee (COREC 07/00404/32).

Provenance and peer review Not commissioned; externally peer reviewed.

Data sharing statement All data collected during this study are stored at the senior author's institution.

\section{REFERENCES}

1. Javaheri S. Sleep disorders in systolic heart failure: a prospective study of 100 male patients. The final report. Int J Cardiol 2006;106:21-8.

2. Vazir A, Hastings PC, Dayer M, et al. A high prevalence of sleep disordered breathing in men with mild symptomatic chronic heart failure due to left ventricular systolic dysfunction. Eur J Heart Fail 2007:9:243-50.

3. Javaheri S, Shukla $R$, Zeigler $H$, et al. Central sleep apnea, right ventricular dysfunction, and low diastolic blood pressure are predictors of mortality in systolic heart failure. J Am Coll Cardiol 2007;49:2028-34.

4. Wang H, Parker JD, Newton GE, et al. Influence of obstructive sleep apnea on mortality in patients with heart failure. J Am Coll Cardiol 2007:49:1625-31.

5. Javaheri S, Caref EB, Chen E, et al. Sleep apnea testing and outcomes in a large cohort of Medicare beneficiaries with newly diagnosed heart failure. Am J Respir Crit Care Med 2010:183:539-46.

6. Hastings PC, Vazir A, O'Driscoll DM, et al. Symptom burden of sleep-disordered breathing in mild-to-moderate congestive heart failure patients. Eur Respir J 2006;27:748-55.

7. Kushida CA, Littner MR, Morgenthaler T, et al. Practice parameters for the indications for polysomnography and related procedures: an update for 2005. Sleep 2005;28:499-521

8. Collop NA, Anderson WM, Boehlecke B, et al. Clinical guidelines for the use of unattended portable monitors in the diagnosis of obstructive sleep apnea in adult patients. Portable Monitoring Task Force of the American Academy of Sleep Medicine. J Clin Sleep Med 2007;3:737-47.

9. Kuna ST, Badr MS, Kimoff RJ, et al. An official ATS/AASM/ACCP/ERS workshop report: research priorities in ambulatory management of adults with obstructive sleep apnea. Proc Am Thorac Soc 2011:8:1-16.

10. Vazir A, Dayer $\mathrm{M}$, Hastings $\mathrm{PC}$, et al. Can heart rate variation rule out sleepdisordered breathing in heart failure? Eur Respir J 2006;27:571-7.

11. Ward NR, Cowie MR, Rosen SD, et al. Detection of sleep-disordered breathing in chronic heart failure patients: utility of heart rate variability versus pulse oximetry? Thorax 2010;65(Suppl 4):A10-11.

12. Ward NR, Roldao V, Cowie MR, et al. Utility of pulse oximetry versus heart rate variability to screen for sleep-disordered breathing in chronic heart failure. $\mathrm{Am} \mathrm{J}$ Respir Crit Care Med 2011;183:A6321.

13. Swedberg K, Cleland J, Dargie $\mathrm{H}$, et al. Guidelines for the diagnosis and treatment of chronic heart failure: executive summary (update 2005): the Task Force for the Diagnosis and Treatment of Chronic Heart Failure of the European Society of Cardiology. Eur Heart J 2005;26:1115-40.

14. Bennett LS, Stradling JR, Davies RJ. A behavioural test to assess daytime sleepiness in obstructive sleep apnoea. J Sleep Res 1997:6:142-5.

15. Iber C, Ancoli-Israel S, Chesson A, et al. The AASM Manual for the Scoring of Sleep and Associated Events: Rules, Terminology and Technical Specifications. 1st edn. Westchester, Illinois: American Academy of Sleep Medicine, 2007.
16. Roche F, Duverney D, Court-Fortune I, et al. Cardiac interbeat interval increment for the identification of obstructive sleep apnea. Pacing Clin Electrophysiol 2002;25:1192-9

17. Task Force of the European Society of Cardiology. Heart rate variability Standards of measurement, physiological interpretation, and clinical use. Task Force of the European Society of Cardiology and the North American Society of Pacing and Electrophysiology. Eur Heart J 1996;17:354-81.

18. Pietzsch JB, Garner A, Cipriano LE, et al. An integrated health-economic analysis of diagnostic and therapeutic strategies in the treatment of moderate-to-severe obstructive sleep apnea. Sleep 2011;34:695-709.

19. Erman MK, Stewart D, Einhorn D, et al. Validation of the ApneaLink for the screening of sleep apnea: a novel and simple single-channel recording device. J Clin Sleep Med 2007; 3:387-92.

20. Vázquez JC, Tsai WH, Flemons WW, et al. Automated analysis of digital oximetry in the diagnosis of obstructive sleep apnoea. Thorax 2000;55:302-7.

21. Guilleminault $\mathbf{C}$, Connolly $\mathrm{S}$, Winkle $\mathrm{R}$, et al. Cyclical variation of the heart rate in sleep apnoea syndrome. Mechanisms, and usefulness of $24 \mathrm{~h}$ electrocardiography as a screening technique. Lancet 1984;1:126-31.

22. Stein PK, Pu Y. Heart rate variability, sleep and sleep disorders. Sleep Med Rev 2012;16:47-66.

23. Szollosi I, Krum H, Kaye D, et al. Sleep apnea in heart failure increases heart rate variability and sympathetic dominance. Sleep 2007;30:1509-14.

24. Leung RS, Floras JS, Lorenzi-Filho G, et al. Influence of Cheyne-Stokes respiration on cardiovascular oscillations in heart failure. Am J Respir Crit Care Med 2003:167:1534-9.

25. Shiomi T, Guilleminault C, Sasanabe R, et al. Augmented very low frequency component of heart rate variability during obstructive sleep apnea. Sleep 1996:19:370-7.

26. Roche F, Celle S, Pichot V, et al. Analysis of the interbeat interval increment to detect obstructive sleep apnoea/hypopnoea. Eur Respir J 2007;29:1206-11.

27. Roche F, Sforza E, Duverney D, et al. Heart rate increment: an electrocardiologica approach for the early detection of obstructive sleep apnoea/hypopnoea syndrome. Clin Sci (Lond) 2004;107:105-10.

28. Damy T, D'Ortho MP, Estrugo B, et al. Heart rate increment analysis is not effective for sleep-disordered breathing screening in patients with chronic heart failure. J Sleep Res 2009;19:131-8.

29. Patel H, Ozdemir BA, Patel M, et al. Impairment of autonomic reactivity is a feature of heart failure whether or not the left ventricular ejection fraction is normal. Int $J$ Cardiol 2010;151:34-9.

30. Fukusaki C, Kawakubo K, Yamamoto Y. Assessment of the primary effect of aging on heart rate variability in humans. Clin Auton Res 2000;10:123-30.

31. Binkley PF, Haas GJ, Starling RC, et al. Sustained augmentation of parasympathetic tone with angiotensin-converting enzyme inhibition in patients with congestive hear failure. J Am Coll Cardiol 1993;21:655-61.

32. Mortara A, La Rovere MT, Pinna GD, et al. Nonselective beta-adrenergic blocking agent, carvedilol, improves arterial baroflex gain and heart rate variability in patients with stable chronic heart failure. J Am Coll Cardiol 2000;36:1612-18.

33. Sforza E, Pichot V, Cervena $K$, et al. Cardiac variability and heart-rate increment as a marker of sleep fragmentation in patients with a sleep disorder: a preliminary study. Sleep 2007;30:43-51

34. Series F, Kimoff RJ, Morrison D, et al. Prospective evaluation of nocturnal oximetry for detection of sleep-related breathing disturbances in patients with chronic heart failure. Chest 2005;127:1507-14.

35. Staniforth AD, Kinnear WJ, Starling R, et al. Nocturnal desaturation in patients with stable heart failure. Heart 1998:79:394-9.

36. Leung RS, Bowman ME, Diep TM, et al. Influence of Cheyne-Stokes respiration on ventricular response to atrial fibrillation in heart failure. J Appl Physiol 2005;99:1689-96.

37. Hogg K, Swedberg K, McMurray J. Heart failure with preserved left ventricular systolic function; epidemiology, clinical characteristics, and prognosis. J Am Coll Cardiol 2004;43:317-27.

38. Bitter T, Westerheide N, Faber L, et al. Adaptive servoventilation in diastolic heart failure and Cheyne-Stokes respiration. Eur Respir J 2009;36:385-92. 